Table 1

Demographic information for DIAN cohort groups.

\begin{tabular}{lllll}
\hline & CDR0 M- & CDR0 M+ & CDR0.5 M+ & CDR1-2 M+ \\
\hline $\mathrm{N}$ & 37 & 44 & 24 & 15 \\
& 25 PS1; 5 PS2; 7 APP & 35 PS1; 3 PS2; 6 APP & 20 PS1; 2 PS2; 2 APP & 13 PS1; 0 PS2; 2 APP \\
Age & $38.9(9.7)$ & $34.6(8.04)$ & $44.5(11.7)$ & $49.3(9.7)$ \\
Estimated Time From Age of Onset & $-7.7(12.1)$ & $-12.4(7.3)$ & $-2.6(8.6)$ & $2.3(8.1)$ \\
\hline
\end{tabular}

older subjects, suggesting network dysconnection may be an early marker of AD-related synaptic failure. The Dominantly Inherited Alzheimer Network (DIAN) cohort offers a unique opportunity to probe $\mathrm{AD}$ related network dysfunction in a much younger cohort, including presymptomatic carriers of presenilin-1 (PS-1), presenilin-2 (PS-2), and amyloid precursor protein (APP) mutations, and to model DMN connectivity as a function of proximity to the observed age of disease onset in these families (time from onset $=$ TFO). Methods: A total of 120 subjects, including 83 mutation carriers (PS-1M+n $=68$; PS-2M+ $\mathrm{n}=5$; АPPM $+\mathrm{n}=10$ ) and 37 non-mutation carriers (M-) from the same families, underwent functional MRI during resting state $(5.3 \mathrm{~min}$ scan). Subjects were then classified into 4 groups based on the Clinical Dementia Rating Scale (CDR) and carrier status (collapsing across mutations; see Table 1 for demographics): CDR0M- $(\mathrm{n}=37)$; CDR0M+ (n $=44)$; CDR 0.5M+ $(\mathrm{n}=24) ; \mathrm{CDR} 1-2 \mathrm{M}+(\mathrm{n}=15)$. Functional connectivity MRI (fc-MRI) analyses were conducted with group-independent component analysis using SPM8 and GIFT. Results: Whole-brain map ANOVA revealed group differences throughout nodes of the DMN, with the strongest effect in the Precuneus/Posterior Cingulate (PPC; $\mathrm{F}(3,116)$ $=14.72, P<0.0001 ;$ Fig-A). Post-hoc comparison showed significantly decreased fc-MRI in asymptomatic CDR0M+ compared to CDR0M- in the PPC $(P<0.05$; Fig-B), right lateral parietal $(P<0.01)$, left lateral temporal $(P<0.0001)$, and medial temporal regions $(P<0.001)$. We observed a negative correlation between DMN connectivity in the PPC and TFO across all mutation carriers $(\mathrm{r}=-.42 ; P<0.001)$, whereas no relationship
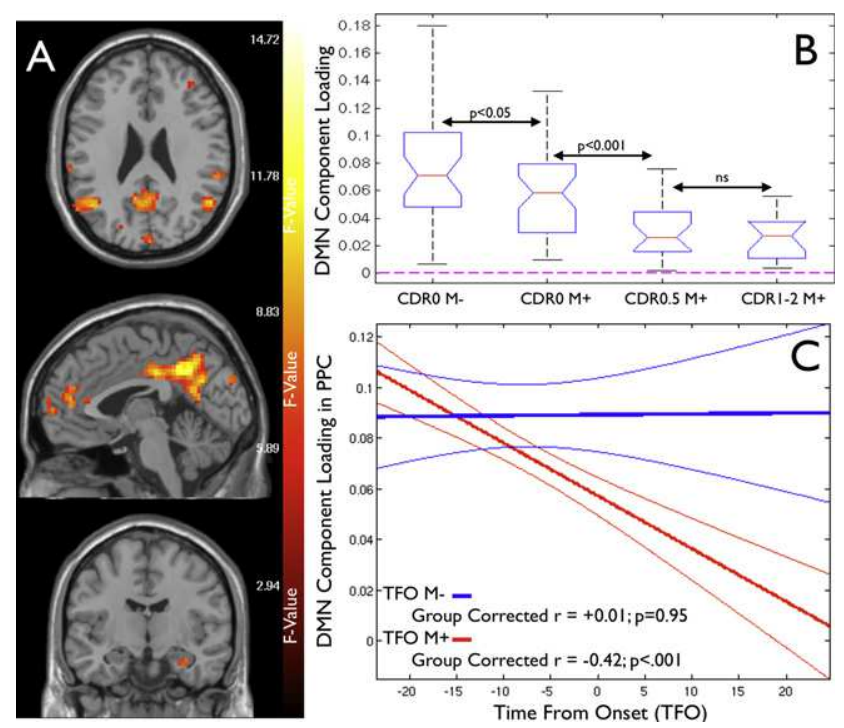

A. Whole brain ANOVA across the 4 groups: threshold $=P<0.001 ; \mathrm{F}=5.8$. B. Box Plots of four groups from the peak voxel in the PPC. C. DMN functional connectivity in the PPC (single voxel) by estimated Time from Age of Onset (Unadjusted data shown). Regression model including CDR as covariate revealed significant differences between $\mathrm{M}$ - and $\mathrm{M}+$ group sloped $(P<0.01)$. was observed among non-carriers $(\mathrm{r}=.01 ; P=0.95)$. The difference in mutation group slopes remained significant when controlling for CDR (difference in slopes $P<0.01$; Fig-C). Conclusions: Impaired connectivity among multiple nodes of the DMN was observed with advancing clinical decline in familial $\mathrm{AD}$, similar to reports in sporadic AD. Presymptomatic mutation carriers demonstrated subtle evidence of DMN dysfunction compared to non-carriers. A strong linear relationship between decreasing DMN integrity and increasing proximity to the expected age of symptom onset suggests that fc-MRI may be useful for tracking disease progression in the preclinical phases of $\mathrm{AD}$.

\section{O2-06-02 FDG METABOLISM IN THE DIAN STUDY OF AUTOSOMAL DOMINANT ALZHEIMER'S DISEASE}

John Becker ${ }^{1}$, Robert Koeppe ${ }^{2}$, Tammie Benzinger ${ }^{2}$, Clifford Jack ${ }^{3}$, Daniel Marcus ${ }^{4}$, Marc Raichle ${ }^{4}$, Paul Thompson ${ }^{5}$, Andrew Saykin ${ }^{6}$, Stephen Correia ${ }^{7}$, Peter Schofield ${ }^{8}$, Christopher Rowe ${ }^{9}$, Nick Fox ${ }^{10}$, Adam Brickman ${ }^{11}$, Bernardino Ghetti ${ }^{6}$, Colin Masters ${ }^{9}$, Chester Mathis ${ }^{12}$, Michael Weiner ${ }^{13}$, Randall Bateman ${ }^{4}$, Anne Fagan ${ }^{4}$, Alison Goate ${ }^{4}$, Chengjie Xiong ${ }^{4}$, Virginia Buckles ${ }^{4}$, Krista Moulder ${ }^{4}$, Richard Mayeux ${ }^{11}$, Ralph Martins ${ }^{14}$, Nigel Cairns ${ }^{4}$, John Ringman ${ }^{5}$, Stephen Salloway ${ }^{15}$, John Morris ${ }^{4}$, Reisa Sperling ${ }^{16}$, Keith Johnson ${ }^{17},{ }^{1}$ Massachusetts General Hospital, Boston, Massachusetts, United States; ${ }^{2}$ University of Michigan, Ann Arbor, Michigan, United States; ${ }^{3}$ Mayo Clinic, Rochester, Minnesota, United States, ${ }^{4}$ Washington University School of Medicine, St. Louis, Missouri, United States; ${ }^{5}$ University of California Los Angeles, Los Angeles, California, United States; ${ }^{\circ}$ Indiana University School of Medicine, Indianapolis, Indiana, United States; ${ }^{7}$ Brown University, Providence, Rhode Island, United States; ${ }^{8}$ University of New South Wales, Sydney, Australia; ${ }^{9}$ University of Melbourne, Melbourne, Victoria, Australia; ${ }^{10}$ University College London, London, United Kingdom; ${ }^{11}$ Columbia University, New York, New York, United States; ${ }^{12}$ University of Pittsburgh, Pittsburgh, Pennsylvania, United States; ${ }^{13}$ University of California San Francisco, San Francisco, California, United States; ${ }^{14}$ Edith Cowan University, Perth, Australia; ${ }^{15}$ Brown University, Providence, Rhode Island, United States; ${ }^{16}$ Brigham and Women's Hospital, Boston, Massachusetts, United States; ${ }^{17}$ MGH HMS, Boston, Massachusetts, United States.

Background: Abnormal brain glucose metabolism has been reported in individuals with genetic risk factors for $\mathrm{AD}$. In order to evaluate the natural history of these changes, participants in the Dominantly Inherited Alzheimer's Network (DIAN) study underwent 18F FDG PET, and we report here an initial assessment of the current baseline FDG-PET DIAN data set. Objective: To evaluate the association of FDG metabolism with age and mutation status in cognitively normal $(C D R=0)$ and symptomatic $(C D R>=0.5)$ individuals. Methods: FDG data sets from 91 CDR0 (47 carriers/44 non-carriers), $20 \mathrm{CDR} 0.5$ (all carriers) and $13 \mathrm{CDR}>=1$ (all carriers) subjects were spatially normalized to an MNI-space template using SPM8, resampled in regions defined by the MNI-based LONI probabilistic atlas, and scaled to cerebellum. Linear regression was used to model the associations of regional average FDG with age, time-to-familial-age-of-onset (TFAO), mutation status and group membership. Results: Compared to CDR0 non- carriers and controlling for age, FDG uptake was significantly lower in CDR0.5 subjects globally $(P<0.01)$ and in AD-vulnerable regions 
including angular gyrus $(P<10-5)$, middle temporal $(P<0.004)$, supramarginal $(P<0.003)$ and precuneus $(P<10-5)$ (Figure 1). Greater decreases in metabolism were seen in $\mathrm{CDR}>=1$ subjects in a similar set of regions $(P$ $<10-5)$. Significant negative associations of FDG metabolism with age, controlling for CDR group, were also seen in these regions $(P<0.04)$. While lower mean regional FDG uptake in CDR0 carriers compared to non-carriers did not reach statistical significance, there was a significant inverse relation between TFAO and carrier FDG uptake measured in an AD-vulnerable aggregate region $(P<0.05)$ and in the angular gyrus $(P<0.05)$ (Figure 2). Conclusions: In symptomatic DIAN participants, FDG metabolism was reduced in a regional pattern similar to sporadic AD. In asymptomatic mutation carriers, a similar pattern of FDG hypometabolism was associated with increasing proximity to their family's median age of symptom onset.

Table

\begin{tabular}{lllll}
\hline & $\begin{array}{l}\text { CDR0 } \\
\text { non-carriers }\end{array}$ & $\begin{array}{l}\text { CDR0 } \\
\text { carriers }\end{array}$ & $\begin{array}{l}\text { CDR0.5 } \\
\text { carriers }\end{array}$ & $\begin{array}{l}\text { CDR }>=1 \\
\text { carriers }\end{array}$ \\
\hline $\mathrm{N}$ & 44 & 47 & 20 & 13 \\
$\mathrm{Sex} \mathrm{M} / \mathrm{F}$ & $19 / 25$ & $17 / 30$ & $10 / 10$ & $7 / 6$ \\
Age & $41.3 \pm 9.0$ & $35.6 \pm 8.6$ & $44.0 \pm 10.8$ & $47.5 \pm 8.6$ \\
$\begin{array}{c}\text { Time to Familial } \\
\text { Age of Onset }\end{array}$ & $-3.9 \pm 12.0$ & $-11.6 \pm 8.0$ & $-0.8 \pm 9.7$ & $1.6 \pm 7.7$ \\
\hline
\end{tabular}

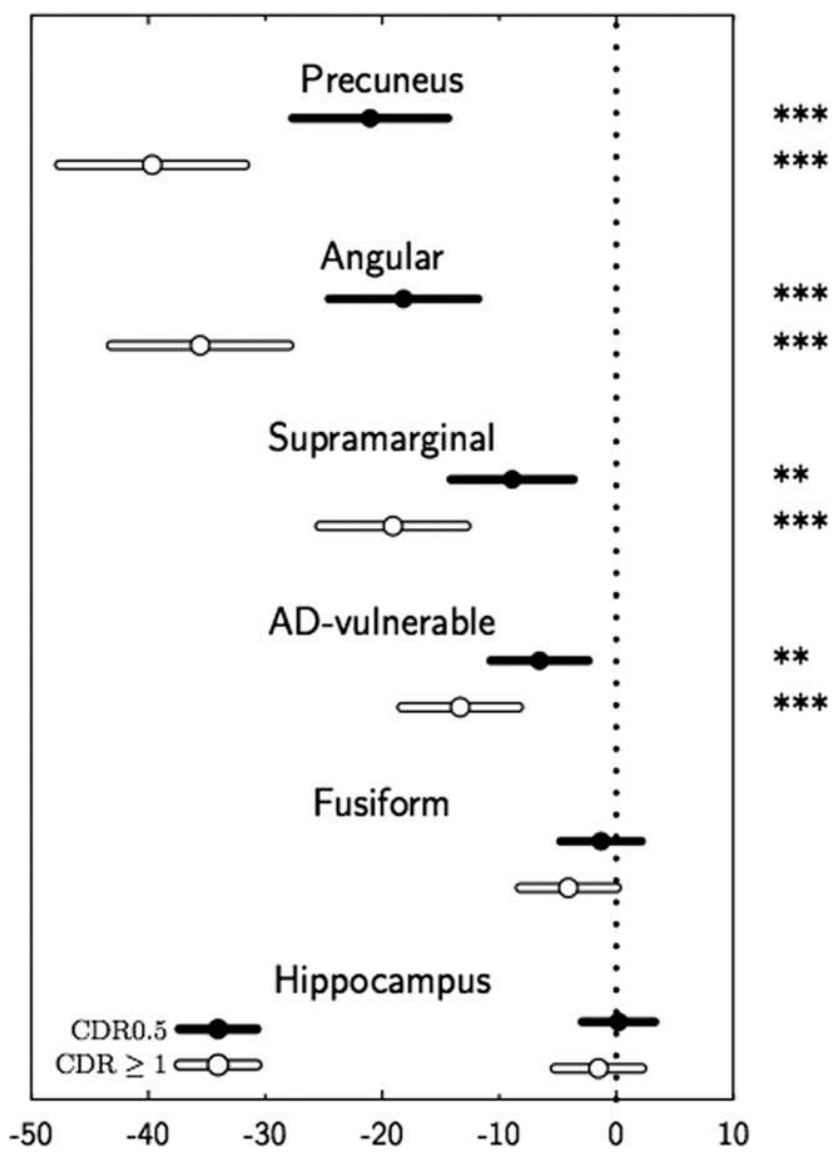

Figure 1. FDG uptake in CDR0.5 and $\mathrm{CDR}>=1$ groups compared to CDR0 non-carriers controlling for age; difference $* 10^{2}$ and $95 \%$ confidence interval. $P$-values: $*(<0.05)$; ** $(<0.01) ; * * *(<0.001)$. AD-vulnerable is an aggregate of regions typically affected in sporadic $\mathrm{AD}$.

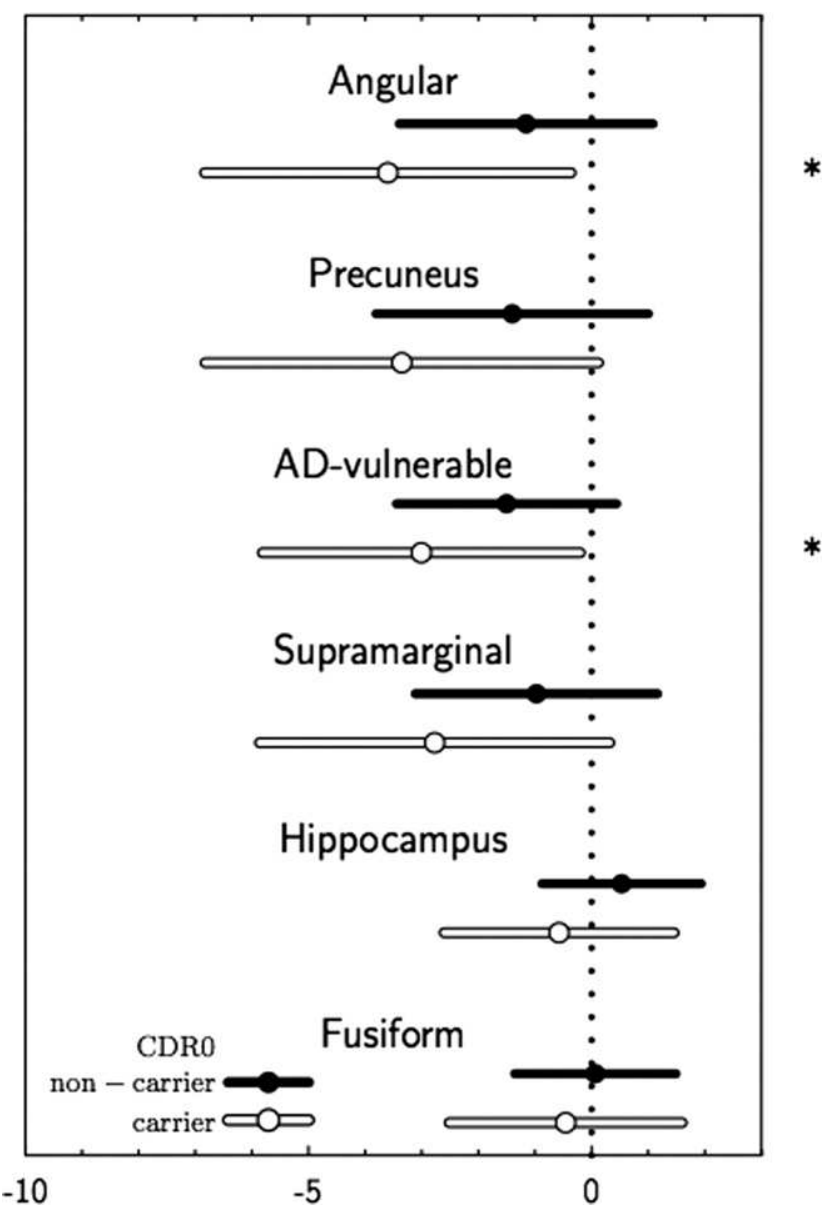

Figure 2. Association of FDG uptake and time-to-familial-age-onset in CDR0 non-carriers and carriers; regression-coefficient* $10^{3}$ and $95 \%$ confidence interval.

\section{O2-06-03 IDENTIFICATION OF NON-INVASIVE SCREENING VARIABLES FOR THE PREDICTION OF AMYLOID ACCUMULATION IN A POPULATION-BASED STUDY OF COGNITIVELY NORMAL ELDERLY INDIVIDUALS}

Michelle Mielke $^{1}$, Heather Wiste ${ }^{1}$, Stephen Wiegand ${ }^{1}$, David Knopman ${ }^{1}$, Val Lowe ${ }^{1}$, Rosebud Roberts ${ }^{1}$, Dana Swenson-Dravis ${ }^{1}$, Bradley Boeve ${ }^{1}$, Yonas Geda ${ }^{2}$, Mathew Senjem ${ }^{1}$, Prashanthi Vemuri ${ }^{1}$, Ronald Petersen ${ }^{1}$, Clifford Jack, Jr, ${ }^{1},{ }^{1}$ Mayo Clinic, Rochester, Minnesota, United States;

${ }^{2}$ Mayo Clinic College of Medicine, Rochester, Minnesota, United States.

Background: The design of secondary Alzheimer's disease (AD) prevention trials in preclinical subjects will likely require documentation of brain amyloidosis for enrollment. The identification of inexpensive and non-invasive screening variables that could predict which individuals have significant amyloid accumulation would reduce screening costs. Methods: 483 cognitively normal $(\mathrm{CN})$ individuals, aged 70-92, from the population-based Mayo Clinic Study of Aging underwent PIB-PET imaging. Logistic regression was used to determine whether age, sex, APOE genotype, family history, or cognitive performance were associated with increased odds of a PIB retention ratio $>1.4$ and $>1.5$. Area under the receiver operating characteristic curve (AUROC) evaluated the discrimination between PIB positive and negative subjects. Positive (PPV) and negative (NPV) predictive value was defined based on an estimated probability $>0.50$ who were PIB-positive. The estimated sample size for each characteristic, by age group (70-79 and 80-89 years), needed to screen to enroll 100 participants 